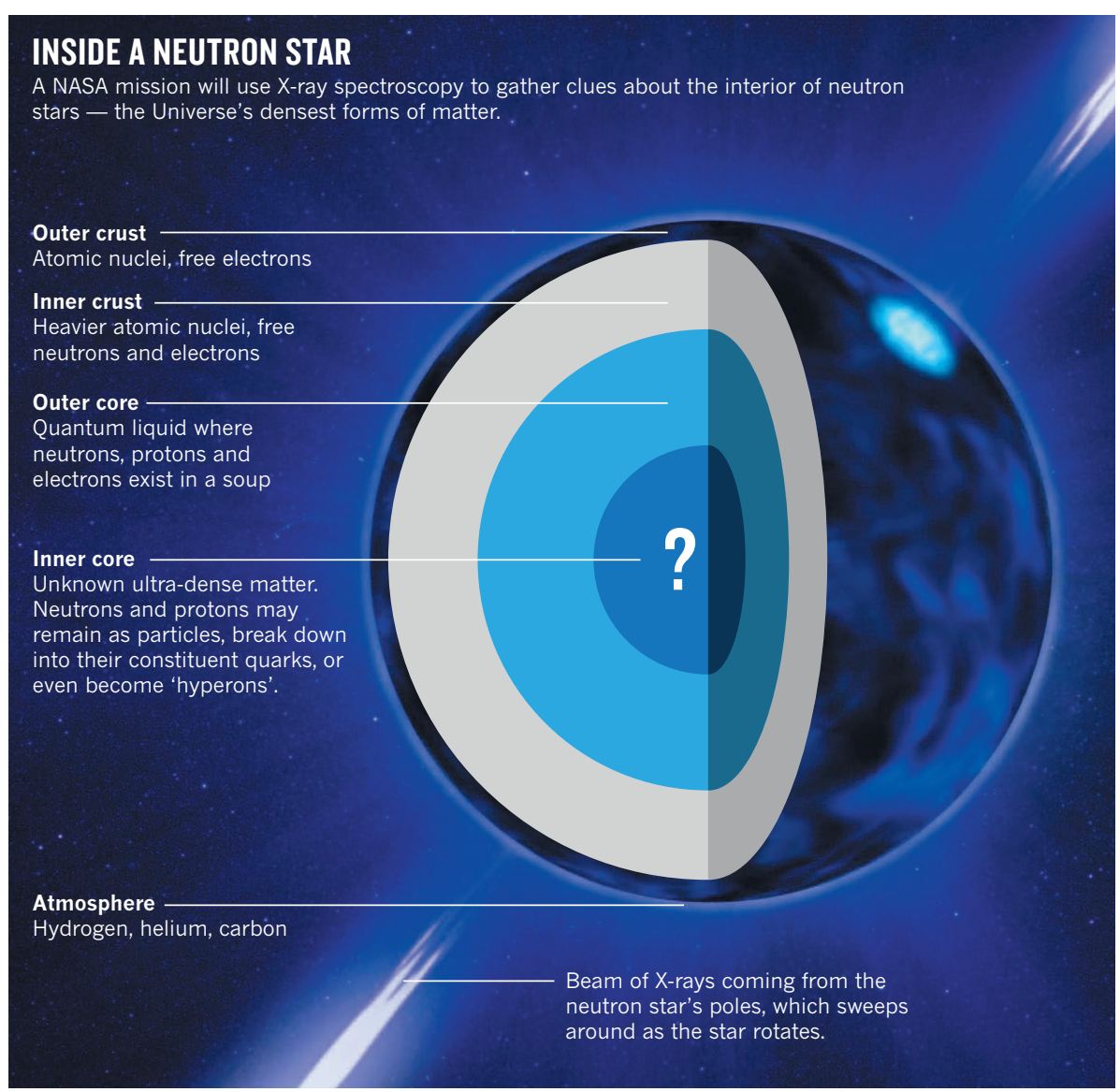

ASTROPHYSICS

\title{
Neutron stars to open their heavy hearts
}

\section{Space mission will peer inside densest matter in the Universe.}

\section{BY ELIZABETH GIBNEY}

$\mathrm{F}$ or half a century, astronomers and physicists have looked at pulsars and asked 'how'? How can something the size of a city pack in more mass than the Sun? How does matter arrange itself to achieve such mind-boggling densities? Answering these questions in the laboratory is impossible. But a space mission due to launch on 1 June could answer some of them. For the first time, astronomers will take a detailed peek into the heavy hearts of these mysterious spinning stars.

"It is a big, big step forward to understand the property of the densest matter in the Universe," says Tetsuo Hatsuda, a theoretical physicist at the RIKEN Interdisciplinary Theoretical and Mathematical Sciences Program in Saitama, Japan. "The state of matter at super high density at the core of the neutron star has been one of the long-standing problems it arrives at the International Space Station, NASA's Neutron Star Interior Composition Explorer (NICER), a washing-machine-sized box, will use X-rays coming from hotspots at the spinning stars' poles to calculate the size of the stars.

Size matters, because a bigger star suggests a stiff core that is relatively able to withstand gravity's compression, which means that it is probably tightly packed with neutrons jostling against each other at higher pressure than that in atomic nuclei. A smaller, more compact star, meanwhile, would mean a soft interior, in which neutrons could be dissolved in a sea of their constituent quarks. Other, more exotic proposals include the core being made of 'hyperons', which incorporate heavier 'strange' quarks within them.

NICER will pinpoint the stars' radii by studying how their huge gravitational fields bend the light they emit. Seen from the space station, the light is fainter when the beam points away, but remains visible because the star's gravitational field diverts some of the light back this way. The extent to which the light dims when the beam faces away tells astronomers about this field, and consequently the star's mass-to-radius ratio. Combined with a mass measurement - derived by studying the $\mathrm{X}$-rays' timing and energy, which are influenced by some of the star's other properties - astronomers can derive the star's size more than twice as accurately as could previous missions.

But to tease apart these complex effects, NICER needs to maximize the photons it collects, channelling them through 56 telescopes designed to observe at the red end of the X-ray spectrum, where many neutron stars are brightest. It then records their arrival time in a silicon detector, with an unprecedented accuracy of better than 100 nanoseconds. "Its timing ability is crazy better than anyone's ever tried before," says Ronald Remillard, an astrophysicist at the Massachusetts Institute of Technology in Cambridge, who will use NICER to observe matter falling into black holes, once the craft has completed its primary pulsar mission.

The mission will also test a new kind of navigation that could allow future spacecraft to pinpoint their location without having to rely on telescopes on Earth. Just as the Global Positioning System uses the ticks of satellites' atomic clocks to triangulate a receiver's position, pulsar navigation would use the arrival times of light from these regularly flashing stars. NICER will probe ten pulsars to test their use in such a system.

If this is successful, NASA's planned Orion space missions may use pulsar-navigation technology as a back-up to conventional navigation, says Keith Gendreau, an astronomer at the Goddard Space Flight Center in Greenbelt, Maryland, and the mission's principal investigator. "We could use pulsars as a way to navigate out into the Solar System and beyond." - 